\title{
Comments on: Confirming geomagnetic Sfe by means of a solar flare detector based on GNSS
}

\author{
Manuel Hernández-Pajares* and Alberto García-Rigo \\ UPC-IonSAT Research Group, Universitat Politècnica de Catalunya, Mod. C3 Campus Nord UPC, c/ Jordi Girona 1, 08034 Barcelona, Spain
}

Received 26 December 2019 / Accepted 10 March 2020

\begin{abstract}
We report two comments affecting the paper "Curto JJ, Juan JM \& Timoté CC, 2019. Confirming geomagnetic Sfe by means of a solar flare detector based on GNSS. J Space Weather Space Clim 9: A42. https://doi.org/10.1051/swsc/2019040": The first comment is the reporting of two mistakes which distorts the central model used for the measurement and detection of solar flares with GNSS, that might affect as well the most part of results and discussions contained in the paper. And the second comment is the clarification about the authors' claim of presenting the first work of using the electron content enhancement estimation at the subsolar point for characterizing solar flares with GNSS data, which is not accurate due to the existence of such previous definition and usage.
\end{abstract}

Keywords: Solar flares / Ionospheric electron content / Global Navigation Satellite Systems

\section{First comment: Mistakes affecting the GNSS Solar Flare model}

In Curto et al. (2019) the main parameter used to analyze the sudden electron content increase of the ionosphere due to solar flares (hereinafter SF) is based on the STEC, defined as Slant TEC (line 3 from the bottom of page 1 of the above-mentioned reference), where TEC is defined as Total Electron Content (lines 4 and 5 of second paragraph of the same page 1). Its variation, an increase during SF in the daylight hemisphere, is expressed in the first equation shown in Section 2.1 "Methodology of Curto et al. (2019)" in terms of the variation of the ionospheric (geometry-free) combination of carrier phases, multiplied by the ionospheric mapping function. This is a first mistake, because such difference of ionospheric carrier phases in the same phase-continuous transmitter-receiver arc, provides directly the STEC variation, without the need of any mapping function term. This is, for example, explicitly indicated in equation (3) of Hernández-Pajares et al. (2012), after neglecting or correcting the small term associated to the wind-up change (see more details in Hernández-Pajares et al. (2011), particularly in Eqs. (17) and (35)).

A second mistake can be seen in the statement at the end of page 1 and the beginning of page 2 in Curto et al. (2019), misinterpreting the meaning of GSFLAI defined in Hernández-Pajares et al. (2012):

\footnotetext{
*Corresponding author: manuel. hernandez@upc. edu
}

The indicator, GNSS Solar Flare Activity Indicator (GSFLAI), was based on the linear fitting of the STEC rates of the satellite-receiver pairs with $\cos \chi$.

The linear dependence, as it is indicated in equations (1) and (2) of Hernández-Pajares et al. (2012), based on Wan et al. (2002), is in terms of the Vertical TEC variation, not the Slant TEC one, as the authors use in the overall paper Curto et al. (2019). In other words, the influence of the GNSS transmitterreceiver line-of-sight slantness in the electron content variation should be removed to formulate the right model, as it is also shown in Figure 1 of Hernández-Pajares et al. (2012).

Summarizing, following the equations and approach described in Curto et al. (2019), it seemed that the authors were multiplying the variation of the ionospheric combination of carrier phases by the ionospheric mapping function, instead of dividing by it, to work on VTEC variations as they should do, due to the first-principles sudden overionization model associated to a solar flare. This overall mistake might strongly compromise the corresponding results and conclusions in such paper.

On the contrary, if the authors of Curto et al. (2019) actually divide the variation of the ionospheric combination of carrier phases by the ionospheric mapping function, then they are actually using the same expression that we used in HernándezPajares et al. (2012) and following works previously referenced, computing actually the VTEC variation, not the STEC variation, at equation (2).

Such VTEC variation expression is an approximation which can content significant errors when very low elevations are 
considered. But this is not the case in our approach due to the usage of a mask of elevation angle above the horizon ( 15 degrees for instance in Hernández-Pajares et al., 2012). Moreover, the usage of the mean value of the mapping function between the consecutive epochs, taking time differences of just $30 \mathrm{~s}$, makes the interpretation as VTEC time difference perfectly suitable. And the last but not the least, we have an indirect proof that this is a very good approximation for the Solar Flare determination with GNSS: the fulfilment of the first principles model of equations (1) and (2) of Hernández-Pajares et al. (2012) based on VTEC rate measurement, when the solar EUV flux rate is derived from such GNSS approach. This is very well correlated with direct solar EUV flux rate measurements from photometers onboard spacecrafts like the SOHO-SEM during one solar cycle (see Hernández-Pajares et al., 2012; Singh et al., 2015).

The potential confusion of Vertical with Slant Electron Content in Curto et al. (2019) should be taken into account to avoid misleading interpretation by any colleague wishing to start working on GNSS solar flare determination, or just wishing to check the results of Curto et al. (2019).

\section{Second comment: Previous definition and usage of subsolar point electron content increase due to solar flares}

We would like to clarify that the approach for detecting and quantifying the Solar Flares with GNSS based on the detrended electron content value on the sub-solar point (SSP) is not new, contrary to what is reported for instance in the paragraph starting in the last line of page 2 of Curto et al. (2019):

Notice that, this is a similar approach to the GSFLAI detector in Hernández-Pajares et al. (2012). However, the GSFLAI measures the slope of the fitting instead of the value of DSTEC at the SSP. Therefore, we can use the DSTEC prediction at the SSP as an indicator of the occurrence of an SF.

Indeed, this is not only similar to the one used in HernándezPajares et al. (2012) - called G1 - and in Singh et al. (2015), but it is strictly the same to the second one defined and used in such paper, called G2, as it is indicated in the following paragraph, at the beginning of page 11 of this reference:

Moreover, a slightly better fitting of G1 with the EUV flux rate has been found in this study, rather than of the second definition G2 (the estimated V-rate at the sub-solar point; shown also in Sect. 3), which justified the main definition of GSFLAI as the solar-zenithal gradient of V-rate.

And the same approach reported as new GNSS Solar Flare index in Curto et al. (2019) has the same foundation (VTEC variability at the subsolar point) to the one used as input during more than one solar cycle in Monte-Moreno \& HernándezPajares (2014), as it can be seen in the abstract of this reference as:
The solar flux variation is modeled as a time series characterized by the subsolar Vertical Total Electron Content double difference in time, computed with dual-frequency GNSS (Global Navigation Satellite Systems) measurements in the daylight hemisphere (GNSS solar flare indicator rate parameter).

Another example of previous definition and usage of GNSS Solar Flare detector based on SSP can be found in García-Rigo (2012), in particular in the following sentences:

The increase of TEC is maximised near the subsolar point but noticeable in most of the whole sunlit ionosphere. ... In case that a solar flare facing the Earth occurs, it is expected that most receiver-satellite rays in the daylight ionosphere will be affected by a simultaneous sudden over-ionization, especially near the subsolar point (i.e., $\left.\mathrm{SZA}=0^{\circ}\right) \ldots$ In the sunlit hemisphere, this relationship approximately follows a cosine function with its maximum at the subsolar point location. ... It can be seen that there is a significant linear trend following the expected cosine function with its maximum at the subsolar point location.

\section{References}

Curto JJ, Juan JM, Timoté CC. 2019. Confirming geomagnetic Sfe by means of a solar flare detector based on GNSS. J Space Weather Space Clim 9: A42. https://doi.org/10.1051/swsc/2019040.

García-Rigo A. 2012. Contributions to ionospheric determination with global positioning system: solar flare detection and prediction of global maps of total electron content, Ph.D. thesis, Universitat Politècnica de Catalunya (UPC), Barcelona, Spain. http://hdl.handle.net/10803/119769.

Hernández-Pajares M, García-Rigo A, Juan JM, Sanz J, Monte E, Aragón-Àngel A. 2012. GNSS measurement of EUV photons flux rate during strong and mid solar flares. Space Weather 10(12): https://doi.org/10.1029/2012SW000826.

Hernández-Pajares M, Juan JM, Sanz J, Aragón-Àngel À, GarcíaRigo A, Salazar D, Escudero M. 2011. The ionosphere: effects, GPS modeling and the benefits for space geodetic techniques. J Geod $\mathbf{8 5}$ (12): 887-907. https://doi.org/10.1007/s00190-011-0508-5.

Monte-Moreno E, Hernández-Pajares M. 2014. Occurrence of solar flares viewed with GPS: Statistics and fractal nature. J Geophys Res Space Phys 119(11): 9216-9227. https://doi.org/10.1002/ 2014JA020206.

Singh T, Hernandez-Pajares M, Monte E, García-Rigo A, OlivaresPulido G. 2015. GPS as a solar observational instrument: Realtime estimation of EUV photons flux rate during strong, medium, and weak solar flares. J Geophys Res Space Phys 120(12): https://doi.org/10.1002/2015JA021824.

Wan W, Yuan H, Liu L, Ning B. 2002. The sudden increase in ionospheric total electron content caused by the very intense solar flare on July 14, 2000. Sci China Ser A Math 45(1): 142. https://doi.org/10.1007/BF02889695. 